J. Lake Sci. (湖泊科学), $2006, \mathbf{1 8}(2): 173-178$

http:// www. jlakes. org. E-mail: jlakes@ niglas. ac.cn

(c) 2006 by Journal of Lake Sciences

\title{
黄河三角洲平原型水库大型底栖动物对磷的积累效应"
}

\author{
王宇庭 ${ }^{1,2}$, 曲 晓 ${ }^{1}$, 张月辉 ${ }^{1}$, 焦晓霞 ${ }^{1}$ \\ $(1$ : 莱阳农学院 水产学院, 青岛 266109) \\ (2: 中国海洋大学, 青岛 266003)
}

\begin{abstract}
摘 要:2003 年春、夏、秋季对黄河三角洲 7 座平原型水库进行了 4 次调查,发现: 1 ) 由摇蚊科幼虫和水蚯蚛构成的大型底 栖动物, 体内磷含量随底泥沉积磷的增加而增加 $(p<0.000)$, 底栖动物对沉积磷的积累系数亦随沉积磷含量的增加而增 加 $(p<0.000) ; 2) 4$ 、 5 月份摇蚊幼虫在底栖动物体所占不同比例 $(20 \%-100 \%)$, 没有显示与底栖动物体内磷含量任何关 系; 3 ) 底栖动物含磷量与水层总磷无显著相关关系; 4$)$ 底栖动物生物量与沉积总磷仅 4 月份有相关 $(p<0.10)$, 其中摇蚊 幼虫生物量与沉积磷量相关显著 $(p<0.05)$, 总体上, 水库底栖动物最高生物量随沉积磷增加而增加; 5 ) 底栖动物生物量 与水层总磷无相关关系, 但去掉底层鱼产量高的水库后, 8 月份二者有显著相关关系 $(p<0.03)$, 且总体底栖动物最高生 物量也随水层总磷增加而增加. 认为: 1) 由摇蚊科幼虫和水蚯蚛构成的大型底栖动物对磷的积累以及摇蚊幼虫的生物量 均与水体磷负荷有密切关系;2) 摇蚊幼虫和水蚯蚂含磷量 (干重) 差异不明显;3) 以摇蚊幼虫为主的底栖动物对磷的积累 主要来自沉积磷的影响, 受季节的影响相对较弱; 4 ) 底栖动物生物量主要受水层总磷的控制, 也受鱼类摄食以及摇蚊幼虫 个体羽化时间不一致的影响.
\end{abstract}

关键词: 平原型水库; 摇蚊幼虫; 水虹蚓;沉积磷;水层总磷

\section{Bioaccumulation of phosphorus by benthic macroinvertebrate in reservoirs at the Yellow River Delta}

\author{
WANG Yuting ${ }^{1,2}$, QU Xiao ${ }^{1}$, ZHANG Yuehui $^{1} \&$ JIAO Xiaoxia ${ }^{1}$ \\ ( 1 : Fishery Dep. of Laiyang Agri. College, Qingdao 266109, P. R. China) \\ (2:Ocean University of China, Qingdao 266003, P. R. China)
}

\begin{abstract}
Benthic invertebrate is a keystone in lake food web to connect nutrients in sediment to animals in and above water, such as fish and waterfowl. Phosphorus is believed to be an important factor leading to eutrophication. Some studies illustrated that the benthos biomass increased with concentration of total phosphorous in water column (TP). The researches on the relationships of phosphorus concentrations in benthic fauna (PIB), lake sediment (PIS) and water column were carried out for 4 times during 2003 in 7 plain shallow reservoirs at the Yellow River Delta. The results displayed: (1) that Chironomidea larvae is the main fauna in the benthos, contributing average $80 \%$ to total benthos biomass, and the other species of Tubificidea to $20 \%$, (2) that PIB had a great significant positive correlation with PIS, but no significant correlation with TP, (3) that the proportion of the larvae has no significant correlation with PIB, (4) that the larvae biomass ( CB) had a significant correlation with PIS only in April during the research, meanwhile, the maximums of CBes increase with the PIS, and (5) that maximums of CBes also increase with TP, while there is a significant correlation between CB and TP if the data of a special reservoir is deleted. The conclusion is: (1) that the phosphorus bioaccumulation by benthic fauna mainly made up of chironomid and tubificid controlled by PIS, while the influence of the fauna constructions and that of the different seasons can be neglected, and (2) that CB is not only controlled by TP, but also influenced by the predation of fish and emergence nonsynchronization of the larvae.
\end{abstract}

Keywords: Bioaccumulation; Chironomidea larvae; Tubificidae; phosphorus in sediment ( PIS), pelagic total 
phosphorus ( TP)

底栖动物作为水域生态系统食物网中腐食链的重要组成成分, 在水生态系统的物质转化和能量流动中 占据重要地位. 磷作为水生植物的重要营养元素, 抑制其有效形态在水体的积累是抑制富营养化的关键. 关 于水生生物对磷积累作用的研究较少 ${ }^{[1]}$, 而关于浅水湖泊 (湿地) 中以摇蚊幼虫为主的底栖动物对磷的积 累作用国内外尚未见报道, 仅见底栖动物中软体动物对重金属的积累作用 ${ }^{[2,3]}$.

黄河磷含量远高于世界河流磷含量的平均值, 其下游 TP 达 $5.6 \mathrm{mg} / \mathrm{L}$, 是长江下游的 30 倍、珠江下游的 80 倍 ${ }^{[4,5]}$. 如此高浓度的磷与黄河三角洲平原型水库水层的 TP 形成反差, 显然河流水层 TP 随泥砂在水库 底质中被沉积起了关键作用一一调查显示沉积磷 ( phosphorus in sediment, PIS) 随着水库使用年限的增加而 增加, 其数值远远高于国内相同水层 $\mathrm{TP}$ 含量湖泊的 $\mathrm{PIS}^{[6]}$. 为了进一步研究 PIS 的动态以及如此高 PIS 的 生态效应, 需要就底栖动物对 PIS 的响应进行研究. 有研究发现其生物量与 TP 相关显著 ${ }^{[7]}$; 而且摇蚊幼虫 能够促进沉积磷向水层的释放 ${ }^{[8]}$. 摇蚊幼虫和水虹蚓是本地水库的主要大型底栖动物, 研究其对磷的积累 作用 (bioaccumulation), 是全面评估其生长、被鱼或水禽摄食等过程对沉积磷影响的基础.

\section{1 材料与方法}

\section{1 样品的采集}

采样时间选择在本地摇蚊幼虫大批羽化期前后的 $4 、 5$ 月, 以及水库中鱼类摄食影响最大的 8 月,和秋 季大量羽化后的 11 月. 底泥样品于 2003 年采自东营市广南水库、孤北水库、孤岛一库、孤岛二库、五号库、 净化站水库和孤东水库等 7 座水库的不同地点 (图 1), 共 55 个样品. 从中留取底泥后, 用 60 目分样䇥将底 栖动物捡出, 并将底栖动物和泥样称湿重后于 $105^{\circ} \mathrm{C}$ 恒温鼓风干燥箱内, 烘干至恒重. 其中 4 、 5 月份的样品 检测所有底栖动物, 即摇蚊幼虫和水蚳蚓; 在分析 $4 、 5$ 月份的数据发现摇蚊幼虫和水虹蚓 (干重) 含磷量的 差异对结构影响不大后, 8、11 月份采集的样品仅收集摇蚊幼虫.

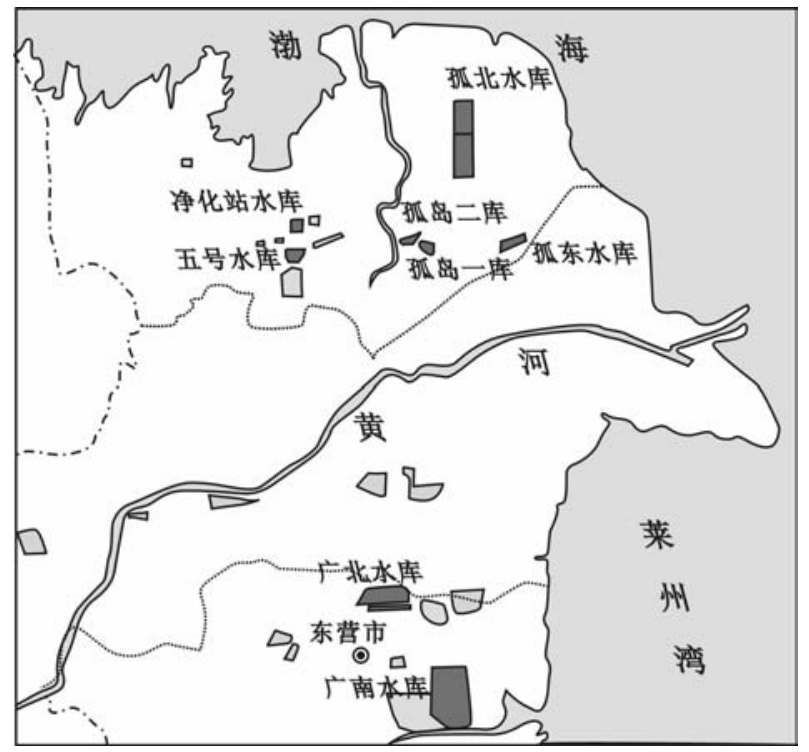

图 1 观测水库于黄河三角洲的位置

Fig. 1 The position of researched reservoirs in the Yellow River Delta

\section{2 化学测定}

(1) 用 $\mathrm{AB} 204-\mathrm{C}$ 型电子天平称取研细的泥样 $0.3000-0.5500 \mathrm{~g}$ 或底栖动物样 $0.0200-0.0500 \mathrm{~g}$ 
(DW), 放于 $250 \mathrm{ml}$ 雉形瓶中, 加 $8 \mathrm{ml}$ 浓 $\mathrm{H}_{2} \mathrm{SO}_{4}$ 和 10 滴 $\mathrm{HClO}_{4}$. 在电炉上加热约 $15 \mathrm{~min}$, 至白烟消失, 样品呈 白色为止. 冷却后定容至 $100 \mathrm{ml}$, 静置过夜.

(2) 取 $5 \mathrm{ml}$ 上清液于 $50 \mathrm{ml}$ 比色管中, 加水至约 $30 \mathrm{ml}$, 加 2 滴 $2,4-$ 二硝基酚, 用 $4 \mathrm{~mol} / \mathrm{L}$ 的 $\mathrm{NaOH}$ 调成 黄色, 再加 $1 \mathrm{~mol} / \mathrm{L} \mathrm{H}_{2} \mathrm{SO}_{4}$ 至黄色刚好褪去. 加人 $1 \mathrm{ml}$ 抗坏血酸摇匀, 反应 $30 \mathrm{~s}$ 后加人 $2 \mathrm{ml}$ 钼酸盐, 摇匀, 静 置显色.

（3）比色:显色 $15 \mathrm{~min}$,用 TU - 1800PC 型紫外分光光度计, 在波长 $880 \mathrm{~nm}$, 用 $30 \mathrm{~mm}$ 比色血比色.

\section{3 数据处理}

回归分析用 SPSS 软件的 11 种回归模型:

(1) 直线: $Y=b_{0}+\left(b_{1} \times X\right)$;

(2) 对数: $Y=b_{0}+\left(b_{1} \times \ln (X)\right)$;

(3) 倒数: $Y=b_{0}+\left(b_{1} / X\right)$;

(4) 二次: $Y=b_{0}+\left(b_{1} \times X\right)+\left(b_{2} \times X^{2}\right)$;

(5) 三次: $Y=b_{0}+\left(b_{1} \times X\right)+\left(b_{2} \times X^{2}\right)+\left(b_{3} \times X^{3}\right)$;

(6) 乘幂: $Y=b_{0} \times X^{b_{1}}$;

(7) 指数: $Y=b_{0} \times b_{1}{ }^{X}$;

(8) $S$ 形曲线: $\ln (Y)=b_{0}+\left(b_{1} / X\right)$;

(9) Logistic 曲线: $Y=1 /\left(1 / u+\left(b_{0} \times\left(b_{1}{ }^{X}\right)\right)\right)$;

(10) 生长方程: $\ln (Y)=b_{0}+\left(b_{1} \times X\right)$;

(11) 双对数: $\ln (Y)=\ln \left(b_{0}\right)+\left(b_{1} \times X\right)$.

从分析结果中选择显著程度最高 ( $p$ 最小) 的回归分析模型.

\section{2. 结果}

\section{1 大型底栖动物的组成及其对磷积累的差异}

调查发现大型底栖动物以摇蚊幼虫和水蚯蚓为主, 4 、5 月份各采样点的摇蚊幼虫湿重占总重的 $20 \%-$ $99 \%$, 平均 $80 \%$ 左右, 其余为水蚯蚓. 摇蚊科 (Chironomidae) 幼虫包括盐生摇蚊 (Chironomus salinarius)、羽摇 蚊 ( C. plumosus)、半折摇蚊 ( C. semireductus)、粗腹摇蚊 (Pelopia. sp.)、红羽摇蚊 ( C. plumosus-reductus) 和塞 氏摇蚊 (C. thummi) 的幼虫; 水生寡毛类为颤蚓科 (Tubificidae) 的水丝蚓属 (Limnodrilus)、尾鳃蚓属 (Branchiura) 和颤蚓属 ( Tubifex).

$4 、 5$ 月份各采样点摇蚊幼虫占底栖动物的比例与底栖动物含磷量 ( phosphorus in benthos, PIB) $(\mathrm{mg} / \mathrm{g}$ (DW) 的回归分析显示, 二者无显著相关关系 (4 月, $p<0.07 ; 5$ 月, $p<0.35$ ).

\section{2 底栖动物含磷量与沉积磷和水层总磷的关系}

$P I S$ 的变动范围为 $1.15,2.72 \mathrm{mg} / \mathrm{g}$, 均值 $2.17 \pm 0.12,(p<0.05) ; P I B$ 的变化范围较 $P I S$ 大, 为 8.12 , $51.0 \mathrm{mg} / \mathrm{g}(\mathrm{DW})$, 均值为 $28.74 \pm 2.93,(p<0.05)$. 不同月份 $P I B$ 与 $P I S$ 的关系如图 2 , 其中 5 月份 $P I B$ 在 $P I S(2.2,2.5)$ 时增速较快, 用 Logistic 曲线回归分析得到, 其最大值为 $61 \mathrm{mg} / \mathrm{g}(\mathrm{DW})$. 将 4 次调查数据合并 (不区分不同季节或不同水库的差异), 二者也存在极显著的相关关系: $P I B=6.87 P I S^{1.80}, R^{2}=0.882, p<$ $0.000 ; \ln P I B=4.81-3.16 / P I S, R^{2}=0.866, p<0.000$.

底栖动物对 PIS 的积累系数 (Condensation index, CI) (PIB/PIS) 变化范围为 $7.06-18.86$,均值 $12.89 \pm$ $0.79,(p<0.05)$, 相关关系为: $C I=6.87 P I S^{0.80}, R^{2}=0.598, p<0.000 ; \ln C I=3.21-1.40 / P I S, R^{2}=0.584, p$ $<0.000$.

PIB 与水层总磷 (TP) 总体没有相关关系 (图 3), 4、8 月份 PIB 与 TP 有相近的变化趋势, 11 月有相反的 变化趋势.

\section{3 底栖动物生物量与 PIS 和 TP 的关系}

4 月份, PIS 与底栖动物生物量 (benthos biomass, BB) ( FW) 无显著相关关系 $(p<0.07)$, 与摇蚊幼虫生 物量 ( chironomid biomass, $\mathrm{CB})(\mathrm{FW})$ 呈显著的相关关系 $\left(C B=1.50 P I S^{2.37}, R^{2}=0.262, p<0.03\right) ; 5$ 月 PIS 与 

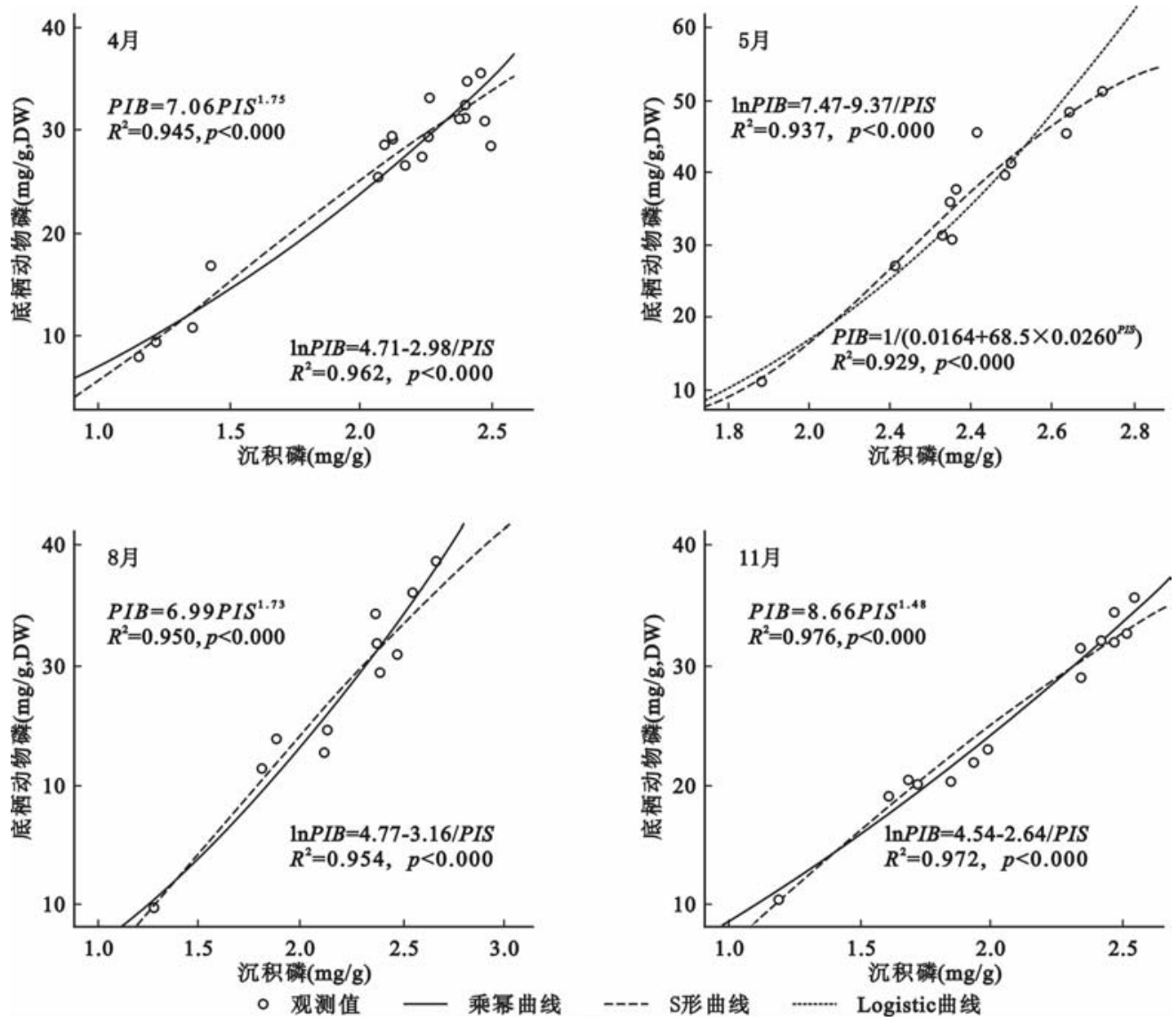

图 2 沉积磷 (PIS) 和底栖动物磷 (PIB) 之间的关系

Fig. 2 Relationship of phosphorus in benthos (PIB) and sediment (PIS)

BB 或 CB 无显著相关关系 $(p<0.61, p<0.38) ; 8$ 月和 11 月 PIS 与 CB 也无显著相关关系 $(p<0.65, p<$ $0.10)$. 将所有沉积磷 (PIS) 和摇蚊幼虫生物量 (CB) 的数据合并, 如图 $4 \mathrm{a}$, 二者无显著的相关关系 $(p<$ 0.28 ), 且数值分布在两条平行线间的区域较少,主要分布在 $C B=1$ 与 $C B=-24+16.7 P I S$ 的区间. 不同月 份水库底栖动物或摇蚊幼虫的平均生物量与平均水层总磷 $(\mathrm{TP})$ 也无显著相关关系 $(p<0.10)$, 如图 $4 \mathrm{~b}$, 且 分布区间也有随 TP 增加的趋势, 并于 3 条直线 $C B=1.0+320 T P 、 C B=-3.5+98 T P$ 和 $C B=-1.0+185 T$ 附近数值分布较多.

为进一步调查底栖动物分布的影响因素,7 月中旬对广南水库围栏内外底栖动物进行了检测: 无鱼的 网拦内的摇蚊幼虫为羽摇蚊,生物量达 $9.5 \mathrm{~g} / \mathrm{m}^{2}$ (与水虾蚓之和的总生物量 $10.2 \mathrm{~g} / \mathrm{m}^{2}$ ), 有鱼的网栏外摇蚊 幼虫为盐生摇蚊,生物量仅 $0.5 \mathrm{~g} / \mathrm{m}^{2}$ (底栖动物以水蚯蚓为主, 总生物量 $5.7 \mathrm{~g} / \mathrm{m}^{2}$ ).

\section{3 讨论}

\section{1 底栖动物对水体磷的积累和稳定作用}

底栖动物以沉积的颗粒有机物 (particulate organic material, POM) 为食, POM 来源于藻类、大型植物碎 片、动物残体和大型动物的粪便. 一方面由于植物对磷有积累作用 ${ }^{[1]}$, 使植物体含磷量受到环境磷含量的 影响, 另一方面 POM 沉积过程中吸附水层和底泥中的磷, 导致 POM 含磷量受到水体磷负荷的影响. 底栖动 
物在不断摄食高磷含量的 POM 后, 可能会因体内磷的排泄速度相对碳、氮等元素的速度较慢, 而在体内积 累. 本调查显示, 底栖动物对磷有积累效应, 其积累系数随着环境中磷含量的升高而升高 $\left(C I=6.87 P I S^{0.80}\right.$, $p<0.000)$. 底栖动物个体对磷的积累过程会因为羽化 (摇蚊幼虫) 或被摄食而终止, 并将体内积累的磷链 释放到水层 (被鱼类摄食) 和直接释放到陆地 (被水禽摄食或直接羽化) 的过程中起重要作用,成为稳定沉 积磷库 (phosphorus pool) 的一个重要生物机制.

调查中发现 8 月和 4 月摇蚊幼虫 (或底栖动物) 生物量各点间差别较大,其中数值较高的可以认为是受 鱼类摄食压力影响较小的,而较 11 月或 5 月下降的幅度可以认为是羽化造成的. 调查中,11月较 8 月摇蚊 幼虫下降最大幅度达到 $25 \mathrm{~g} / \mathrm{m}^{2}, 5$ 月较 4 月达 $10 \mathrm{~g} / \mathrm{m}^{2}$. 以本调查中测定的摇蚊幼虫干物质含量 $15 \%$, 含磷 量按 $30 \mathrm{mg} / \mathrm{g}(\mathrm{dw})$ 计算, 则摇蚊幼虫一年 2 次集中羽化可将底泥中 $160 \mathrm{mg} / \mathrm{m}^{2}$ 磷释放到水层和陆地; 如果 5 、 11 月份的摇蚊幼虫均为新发生的个体, 这个数值达到 $230 \mathrm{mg} / \mathrm{m}^{2}$, 相当于 $6 \mathrm{~m}$ 水深磷 $0.03-0.04 \mathrm{mg} / \mathrm{L}$ 的沉 积量.

\section{2 影响底栖动物积累磷的因素}

所有采样点数值合并分析显示 PIB 与 PIS 呈极显著的相关关系, 说明不同季节和不同采样点对底栖动 物积累磷的效应 (主要来自 PIS) 影响都不大 (图 2)．4、8、11 月 PIB 与 PIS 的两种回归方程形式相同、系数 接近, 其中 $S$ 形曲线回归方程近似为 $\ln P I B=5-3 / P I S$; 仅 5 月份 PIB 随 PIS 增速较快, 最显著的相关关系形 式是 S 形曲线和 Logistic 曲线. 因而, 摇蚊幼虫对磷的积累作用主要与沉积磷的丰度 (PIS)有关,而季节的影 响相对较弱,这与河蚬对重金属的积累作用相似 ${ }^{[3]} .5$ 月份的这种可能情况是春季 POM 含磷量较高, 而且 PIS 较高处低龄幼虫较多, 摄食量较大造成的.

$\mathrm{PIB}$ 与水层 TP 总体上没有相关关系 (图 3)，4、8 月份二 者有相同的变化趋势, 11 月有相反的变化趋势. 4、8 月份风 浪较小, 风浪搅动对 PIS 与 TP 平衡关系影响较弱, 使 TP 和 PIS 一样与 PIB 呈相同的变化趋势; 11 月风浪较大,受影响大 的水库, DIS 再悬浮 (resuspend) 较多, TP 较高, 使得此时二者 变动趋势相反, 因而 DIP 也与 TP 成相反走势.

$4 、 5$ 月份摇蚊幼虫所占底栖动物比例与底栖动物平均磷 含量 (PIB) 的相关分析显示, 二者不呈显著的相关关系. 由于 摇蚊所占生物量的比例为 $20 \%-99 \%$, 而 PIB 与沉积磷 (PIS) 呈极显著的相关关系, 因而可以推测以摇蚊幼虫为主 的摇蚊幼虫和水蛏蚓组成的大型底栖动物群落, 积累磷的效 应主要受沉积磷 (PIS) 影响, 而受底栖动物本身群落结构的 影响较小.

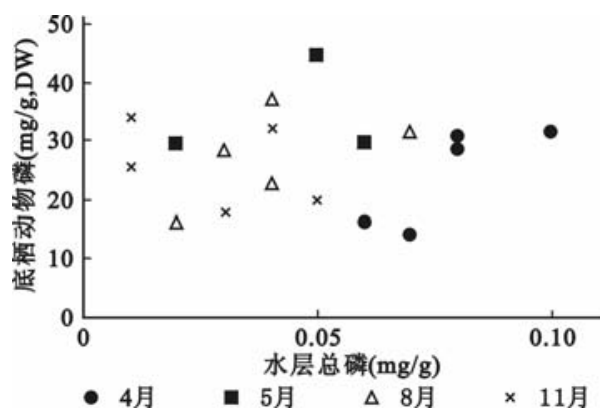

图 3 底栖动物磷与水层总磷之间的关系

Fig. 3 Relationship of phosphorus in

benthos (PIB) and water ( TP)

\section{3 底栖动物生物量与 PIS 和 TP 的关系}

4 月份底栖动物生物量 (BB) 特别是摇蚊幼虫生物量 (CB) 随沉积磷 (PIS) 含量的增高而增加, 其它月份没有这样的相关关系. 图 $4 \mathrm{a}$ 显示, 在 $1.0<C B<(-24+$ 16.7PIS ) 区间数值分布比较密集, 各月份、各水库不同样点的数值都有分布, 而在 $(-24+16.7 P I S)<C B<$ $(-9.0+16.7 P I S)$ 比较稀疏, 仅 4.8 月份部分水库的数值有分布, 可能是没有受到鱼类摄食压力而且在摇 蚊幼虫羽化前的最大生物量状态 $4 、 5$ 月份的底栖动物生物量与 TP 无相关关系, 所有采样时间 CB 与 TP 也 无显著相关关系, 但在 8 月份, 如果将半精养河蟹的五号水库去除, 其它水库的 CB 与 TP 相关显著 $(p<$ 0.02 ). 总体分布趋势如图 4b, 其中从上至下的 3 条直线,第一条线周围以 8 月份的点为数值, 第三条线没 有 8 月份的数值, 而第二条线周围有所有 4 次采样的数值. 结合 7 月份调查的广南水库围网内外的结果可 以推测, 底栖动物的分布除受到底泥营养状态的影响, 也受到鱼类摄食压力的影响, 而且摇蚊幼虫相对水蚯 蚓可能受到更大的摄食压力.

显然, 相对于底栖动物的含磷量, 以摇蚊幼虫为主的底栖动物现存量影响因素更多. 总体趋势受到水体 磷负荷的影响,但 TP 比 PIS 的影响可能更大:TP 决定水层有机物的合成能力, 决定底栖动物的饵料丰度, 

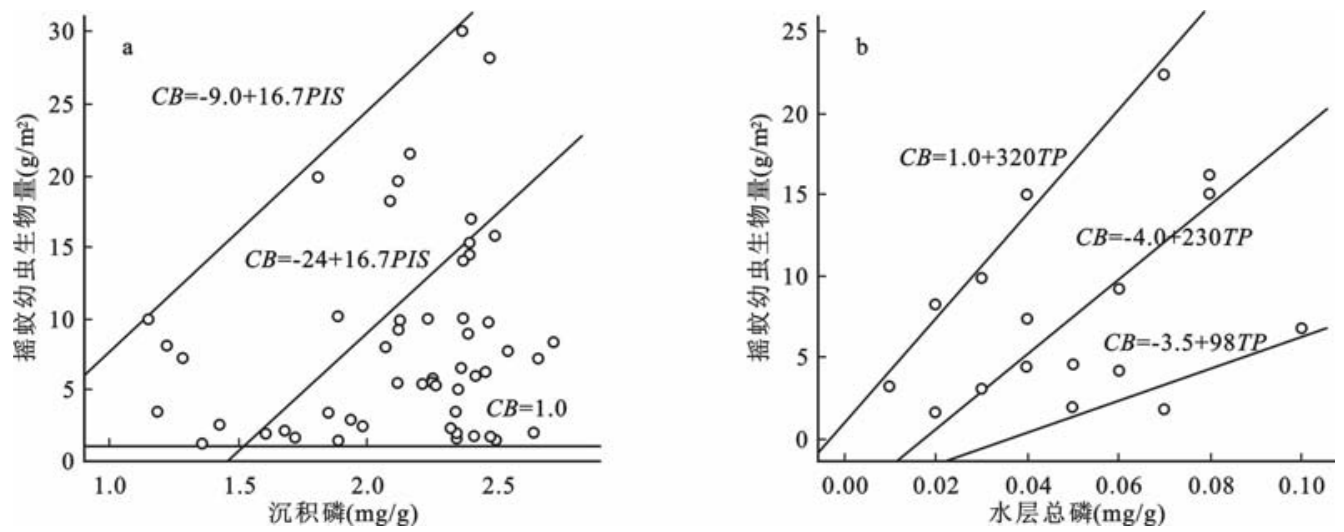

图 4 底泥磷 (PIS) 或水层总磷 (TP) 与摇蚊幼虫生物量 $(\mathrm{CB})$ 之间的关系

Fig. 4 Relationship of between Chironomide larvae biomass (CB) and deposited phosphorus (PIS) or epigic total phosphorus (TP)

而 PIS 决定最终被底栖动物摄食饵料的含磷量. 另外,鱼类摄食压力的不均匀以及摇蚊幼虫个体间发育的 不同步, 导致了同一时间不同采样点数值的差别. 陈其羽等 ${ }^{[7]}$ 调查的武汉东湖底栖动物的生物量与不同采 样点营养物质丰度有关的结果可能是在鱼 (特别是底层鱼类) 现存量较少的情况下得到的, 而 Leppae M 等 ${ }^{[9]}$ 鱼类影响底栖动物现存量和群落结构的结果是在鱼类现存量较高情况下得到的.

\section{4 结语}

以摇蚊幼虫为主的摇蚊幼虫和水蚯蚓构成的底栖动物群落对磷有明显的富集效应, 摇蚊幼虫和水蚯蚓 富集磷的效应差别不明显,磷的来源主要是沉积磷. 群落生物量也受到水体磷负荷的控制, 主要来自水层总 磷, 鱼类摄食和摇蚊幼虫羽化的不同步也对群落生物量构成影响. 在忽略鱼类摄食影响的情况下, 摇蚊幼虫 羽化每年可将底泥磷 $160-230 \mathrm{mg} / \mathrm{m}^{2}$ 释放到水层和陆地.

\section{5 参考文献}

[1] 郁建栓, 戴树桂, 陈甫华. 天然湖水表面微层砷、磷酸盐、悬浮颗粒及藻类富集现象的研究. 环境化 学, $1997,16(4)$ : $359-363$.

[2] 张少娜, 孙 耀, 宋云利. 紫贻贝 (Mytilus edulis) 对 4 种重金属的生物富集动力学特性研究. 海洋与 湖沼,2004,35(5):411-416.

[3] 孙平跃, 王 斌. 季节变化和个体大小对河蚬积累重金属的影响. 海洋通报,2004,23(2):19-24.

[4] 段水旺, 章 申. 中国主要河流控制站氮、磷含量变化规律初探. 地理科学, 1999, 19(5): 411 -416 .

[5] Meybeck M. Carbon, nitrogen and phosphorus transport by world rivers. Am J Sci, 1982, 282 :401 - 450.

[6] 王宇庭, 曲明敏, 任志勇 等. 黄河三角洲平原型水库总磷的变动趋势. 中国海洋大学学报 (自然科 学版), 2004, 34(1):37-42.

[7] 陈其羽, 梁彦龄, 吴天惠. 武汉东湖底栖动物群落结构和动态的研究. 水生生物学集刊, 1980, 7 (1) : $42-55$.

[8] 陈天乙, 刘 孜. 摇蚊幼虫对底泥中氮磷释放作用的研究. 昆虫学报, 1995, 38(4) : 448-451.

[9] Leppae M, Haema Elaeinen H, Karjalainen J. The response of benthic macroinvertebrates to whole-lake biomanipulation. Hydrobiologia, 2003, 498(1-3) : $97-105$. 